\title{
Jahresinhaltsverzeichnis 69. Jahrgang 2021
}

Editorial

$1 / 3,2 / 213$

Luise Anter

Mein Text, meine Meinung, meine Wissenschaftlerin? Eine qualitative Untersuchung zur Nutzung von Wissenschaftler*innen als opportune Zeugen ...

Karin van Es / Mirko Tobias Schäfer / Maranke Wieringa

Tool Criticism and the Computational Turn: A „Methodological Moment" in Media and Comunication Studies

Stefan Gürtler

Audience Gatekeeping in der Wirtschaftskommunikation: der Fall Greensill ...

$4 / 528$

Mario Haim

Gütekriterien und Handlungsempfehlungen für die Entwicklung von

Forschungssoftware in der Kommunikations- und Medienwissenschaft

$1 / 65$

Andreas Hepp / Susan Benz / Piet Simon

Zwischen Utopie und Dystopie. Oder: Wie der öffentliche Diskurs über die Maker- und Quantified-Self-Bewegung in Deutschland und Großbritannien die

Pioniergemeinschaften zu Treibern tiefgreifender Mediatisierung macht ...

Andreas Hepp / Wiebke Loosen / Hendrik Kühn / Leif Kramp / Paul Solbach

Die Figuration des Pionierjournalismus in Deutschland: Akteure und

Experimentierbereiche

Andreas Hepp / Wiebke Loosen / Uwe Hasebrink

Jenseits des Computational Turn: Methodenentwicklung und Forschungssoftware in der Kommunikations- und Medienwissenschaft - zur Einführung in das Themenheft .

Olaf Hoffjann

Stigmatisierte Pressesprecher und Werberinnen?

Olaf Hoffjann / Lucas Seeber

Auf dem Weg in die postfaktische Politik?

Florian Hohmann

Co-Creation als Entwicklungsmethode. Zu Möglichkeiten und Grenzen partizipativer Forschungssoftwareentwicklung am Beispiel der Sortiersoftware MeSort und

Tagebuchsoftware MeTag

Erik Koenen

Forschungssoftware für die Kommunikations- und Mediengeschichte.

Epistemologische Herausforderungen und Perspektiven

Laura Leißner

Die Digitalisierung von Lebensstilpolitik. Wie Soziale Medien lebensstilpolitisches

Engagement prägen 
Stephan Niemand

Mediatisierte Lebensführung und ihr Wandel durch Alltagsumbrüche. Zur Relevanz von Übergangsphasen in der Rezeptionsforschung

Paula Nitschke / Moritz Schweiger

Alltagspraktiken digitaler Teilhabe in Nachbarschaft und Stadt

Gerret von Nordheim / Lars Koppers / Karin Boczek / Jonas Rieger / Carsten Jentsch / Henrik Müller / Jörg Rahnenführer

Die Entwicklung von Forschungssoftware als praktische Interdisziplinarität . . .

Anne Reif / Evelyn Peter / Tamie Gillner / Lisa-Marie Hortig / Alexander Joost / Monika Taddicken

Vom Bildschirm auf die Straße? Eine empirische Untersuchung der Identifikation, Online-Partizipation und des Klimaproblembewusstseins von Fridays-for-Future-

Beteiligten

Richard Rogers

Digitale Methoden: Zur Positionierung eines Ansatzes

Johanna Schäwel / Regine Frener / Philipp K. Masur / Sabine Trepte

Learning by doing oder doing by learning? Die Wechselwirkung zwischen Online-

Privatheitskompetenz und Datenschutzverhalten

Christian Strippel

Forschungsinfrastrukturen für die Kommunikations- und Medienforschung im

deutschsprachigen Raum. Initiativen, Bedarfe und Perspektiven

Reihe „Methodeninnovationen in der Kommunikationswissenschaft“

Nils Jungmann / Michael Strothoff / Olaf Jandura

Jenseits fixer Lagemaße. Die Change-Point-Analyse als Mittel zur empirischen

Einteilung von Gruppen

Carmen Koch / Nadine Klopfenstein Frei / Iris Herrmann

Das Smartphone-Tagebuch in der Mediennutzungsforschung. Ein qualitativer

Methodenansatz unter der Lupe

Literatur

Besprechungen

Jan-Hinrik Schmidt

Martin Andree / Timo Thomsen (2020): Atlas der digitalen Welt. Frankfurt/New York: Campus

Jamie Medhurst

Klaus Arnold / Paschal Preston / Susanne Kinnebrock (Hrsg.) (2020): The Handbook of European Communication. New York: Wiley

Ole Kelm

Chris Bail (2021): Breaking the Social Media Prism. How to Make Our Platforms Less

Polarizing. Princeton \& Oxford: Princeton University Press 


\section{Christoph Classen}

Thomas Birkner / Patrick Merziger / Christian Schwarzenegger (Hrsg.) (2020): Historische Medienwirkungsforschung. Ansätze, Methoden und Quellen. KöIn:

Herbert von Halem

Nicola Döring

Cornelia Brantner / Gerit Götzenbrucker / Katharina Lobinger / Maria Schreiber (Hrsg.) (2020): Vernetzte Bilder. Visuelle Kommunikation in Sozialen Medien. Köln: Halem . .

Joan Kristin Bleicher

Hans-Jürgen Bucher (Hrsg.) (2020): Medienkritik. Zwischen ideologischer

Instrumentalisierung und kritischer Aufklärung. Köln: Halem

Walter Hömberg

Jens Malte Fischer (2020): Karl Kraus. Der Widersprecher. Biografie. Wien: Paul Zsolnay

Thomas Horky

Kirsten Frandsen (2020): Sport and Mediatization. Milton Park, New York: Routledge.

Andreas Schulz

Christian Fuchs (2021): Soziale Medien und Kritische Theorie. Eine Einführung. 2 Aufl. München: UVK

\section{Christian Pentzold}

Stephan O. Görland (2020): Medien, Zeit und Beschleunigung. Mobile Mediennutzung in Interimszeiten. Wiesbaden: Springer VS

Margreth Lünenborg

Heike Haarhoff (2020): Nafris, Normen, Nachrichten. Die Standards journalistischer Berichterstattung am Beispiel der Herkunftsnennung mutmaßlicher Straftäter der Kölner Silvesternacht 2015/2016. Baden-Baden: Nomos

\section{Edgar Lersch}

Michael Haller / Walter Hömberg (Hrsg.) (2020): „Ich lass mir den Mund nicht verbieten!" Journalisten als Wegbereiter der Pressefreiheit und Demokratie.

Ditzingen: Philipp Reclam jun.

Johannes Schmees

Matthias Herdegen / Johannes Masing / Ralf Poscher / Klaus F. Gärditz (Hrsg.) (2021): Handbuch des Verfassungsrechts. Darstellung in transnationaler Perspektive.

München: C. H. Beck

Martin Fertman

Otfried Jarren / Christoph Neuberger (Hrsg.) (2020): Gesellschaftliche Vermittlung in der Krise. Medien und Plattformen als Intermediäre. Baden-Baden: Nomos ...

Gerhard Vowe

Andreas Jungherr / Gonzalo Rivero / Daniel Gayo-Avello (2020): Retooling Politics. How Digital Media are Shaping Democracy. Cambridge: Cambridge University Press ....... 
Daniel Süss

Michaela Kramer (2020): Visuelle Biografiearbeit. Smartphone-Fotografie in der

Adoleszenz aus medienpädagogischer Perspektive. Baden-Baden: Nomos

Joachim Westerbarkey

Uwe Krüger / Sebastian Sevignani (Hrsg.) (2020): Ideologie, Kritik, Öffentlichkeit. Verhandlungen des Netzwerks Kritische Kommunikationswissenschaft. Frankfurt/ Main: Westend

Joan Kristin Bleicher

Margreth Lünenborg / Claudia Töpper / Laura Sūna / Tanja Maier (2021): Affektive Medienpraktiken. Emotionen, Körper, Zugehörigkeiten im Reality TV. Wiesbaden: Springer

Andreas Hepp

Denis McQuail / Mark Deuze (2020): McQuail's Media and Mass Communication

Theory, $7^{\text {th }}$ edition. London: Sage

Ingrid Paus-Hasebrink

Stephan Niemand (2020): Alltagsumbrüche und Medienhandeln. Eine qualitative Panelstudie zum Wandel der Mediennutzung in Übergangsphasen. Wiesbaden:

Springer VS

Niels Borchers

Ole Nymoen / Wolfgang M. Schmitt (2021): Influencer. Die Ideologie der Werbekörper. Berlin: Suhrkamp

Oliver Zöllner

Claudia Paganini (2020): Werte für die Medien(ethik). Baden-Baden: Nomos

Melanie Radue

Carola Richter / Claudia Kozman (Hrsg.) (2021): Arab Media Systems. Open Book

Publishers...

Joan Kristin Bleicher

Christian Richter (2020): Fernsehen - Netflix - Youtube. Zur Fernsehhaftigkeit von On-

Demand-Angeboten. Bielefeld: transcript

Christoph Raetzsch

Simon Strick (2021): Rechte Gefühle. Affekte und Strategien des digitalen Faschismus.

Bielefeld: transcript

Gerhard Vowe

Michael Tomasello (2020): Mensch werden. Eine Theorie der Ontogenese. Berlin:

Suhrkamp

Michael Haller

Mandy Tröger (2019): Pressefrühling und Profit. Wie westdeutsche Verlage 1989/1990 den Osten eroberten. Köln: Herbert von Halem

Kai-Uwe Hugger

Christine Trültzsch-Wijnen (2020): Medienhandeln zwischen Kompetenz, Performanz und Literacy. Wiesbaden: Springer VS 
Lothar Mikos

Hedwig Wagner (Hrsg.) (2020): Europäische Medienwissenschaft. Zur Programmatik

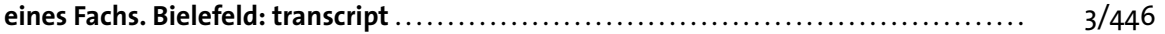

Jörg Requate

Michael Wild (2020): Öffentlichkeit in unsicheren Zeiten. Zur Analyse öffentlicher

Kommunikation in Revolutionen. Köln: Herbert von Halem .................... 2/331

Uwe Krüger

Jennifer Wladarsch (2020): Metakommunikation und die Qualität des Journalismus.

Einfluss von Metakommunikation auf Qualitätserwartungen und -bewertungen bei

Nachrichtennutzern im Internet. Baden-Baden: Nomos

Zeitschriftenlese $.1 / 174,2 / 335,3 / 449,4 / 608$

Literaturverzeichnis

$.1 / 196,2 / 355,3 / 473,4 / 629$

Dissertationsübersicht . . . $.1 / 199$

Hinweise für Autorinnen und Autoren $.1 / 207,2 / 358,3 / 479,4 / 631$ 